\title{
Micro-Mechanical In Situ Measurements in Thin Film Systems Regarding the Determination of Residual Stress, Fracture Properties and Interface Toughness
}

\author{
R. Konetschnik ${ }^{1}$, D. Kiener ${ }^{1}$, D. Kozic ${ }^{2}$, H.-P. Gänser ${ }^{2}$ and R. Brunner ${ }^{2}$ \\ 1. Department Materials Physics, Montantuniversitaet Leoben, Leoben, Austria \\ 2. Materials Center Leoben Forschung GmbH, Leoben, Austria
}

The major trend in microelectronics industry is driven by miniaturization and the increase in functionality [1]. According to root cause analysis from determined failures it is often found that failures in microelectronic components are often triggered by various thermal and mechanical loadings during industrial manufacturing processes like the backend of line (BEOL) processes. Here, single- and/or multiple metal layers or structures are deposited mainly on Si-substrates. These processes, due to the combination of different materials, temperature and topologies, cause residual stresses and may induce defects, which affect interface integrity and eventually cause failures of the device during application. This heavily concerns for example 3D integrated microelectronic devices, power electronics, micromechanical devices, sensors, etc. However, it is a great challenge to understand (1) the complex interplay between thin film combinations, (2) resulting residual stresses, (3) interface properties and (4) fracture behavior.

In this work we consider a toolbox for micro-mechanical testing suitable to provide information regarding material properties of metallic single- and multiple-thin films on an $\mathrm{Si}$ (100) substrate. The measurement of residual stresses, miniaturized fracture and interface toughness properties are performed by using in situ SEM experiments.

For the investigations we use four different film system configurations namely (1) Cu, (2) W-W, (3) W$\mathrm{Cu}-\mathrm{W}$ and (4) $\mathrm{Cu}-\mathrm{W}-\mathrm{W}$. The individual films show a thickness of about $500 \mathrm{~nm}$. All samples were deposited at room temperature on a single crystalline Si (100) with a thickness of $525 \mu \mathrm{m}$ by using a Mantis Sputter System. Three different sputtering targets $(\mathrm{Cr}, \mathrm{Cu}, \mathrm{W})$ were used. Before the $\mathrm{Cu}$ or $\mathrm{W}$ layers were sputtered on the $\mathrm{Si}$, an about $10 \mathrm{~nm}$ thick $\mathrm{Cr}$ - layer was deposited as a seed layer on top of the $\mathrm{Si}(100)$ substrate. Samples for the micro-mechanical testing were prepared in a first stage by using a Hitachi-E3500 Cross Section Polisher. The samples were coated prior to the milling with a lacquer film to avoid breaking. In the second step the samples are prepared by focused ion beam (FIB) milling using a LEO 1540XB Zeiss FIB workstation. The residual stress measurements were carried out on a Zeiss AURIGA-CrossBeam workstation (Zeiss, Oberkochen). Here, the so called ion removal method (ILR) is applied on the prepared micro-beam. The beam is cut free on one side and gradually thinned in $50 \mathrm{~nm}$ steps on the other side within the defined ILR area. The deflection of the micro-beam is then related to the occurring residual stresses within the thin film system and can be calculated from the measured deflection and geometry of the beam by using analytical or numerical calculations [2]. All double cantilever beam (DCB) tests for the interface toughness measurements and fracture experiments were performed in-situ in a LEO 982 SEM using an UNAT-SEM indenter [3].

The residual stress profiles could be determined locally with a depth resolution of $50 \mathrm{~nm}$ for the four different thin film systems. We discuss the application and argue that the in situ method is highly suitable for the local determination of the residual stress and stress gradient in thin film systems. With respect to the in situ fracture experiments we discuss the impact of the elastic properties and residual 
stress gradients on the fracture behavior of the thin films and connected possibilities. For the interface toughness measurements we discuss possibilities by using the micro-DCB tests. We conclude that the choice of the proper micro-mechanical test for the characterization of thin films is highly crucial and strongly depends on the material of interest, thin film configuration and interface properties [4].

\section{References:}

[1] W. Arden, et al., 'More-than-Moore` White Paper, "International Technical Roadmap for Semiconductors, 2010.

[2] R. Treml, et al., Acta Materialia 103, (2016), p. 616.

[3] R. Treml, et al., Extreme Mechanics Letters 8 (2016), p. 235.

[4] Financial support from the Austrian Federal Government (in particular from Bundesministerium für Verkehr, Innovation und Technologie and Bundesministerium für Wissenschaft, Forschung und Wirtschaft) represented by Österreichische Forschungsförderungsgesellschaft mbH and the Styrian and the Tyrolean Provincial Government, represented by Steirische Wirtschaftsförderungsgesellschaft mbH and Standortagentur Tirol, within the framework of the COMET Funding Programme (No. 837900) is also gratefully acknowledged.

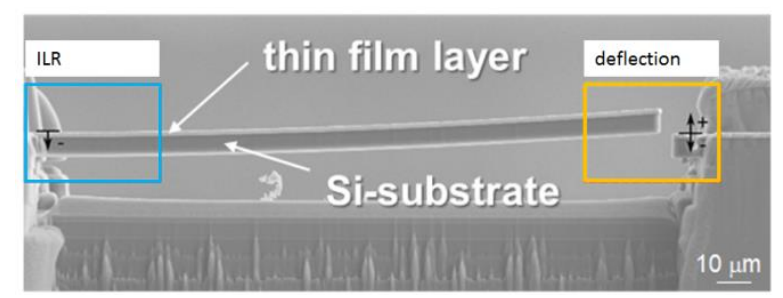

Figure 1. SE micrograph which shows the micro-beam used for the local determination of the residual stress. Left side: ILR area where the gradual layer removal occurs. Right side: deflection is measured.

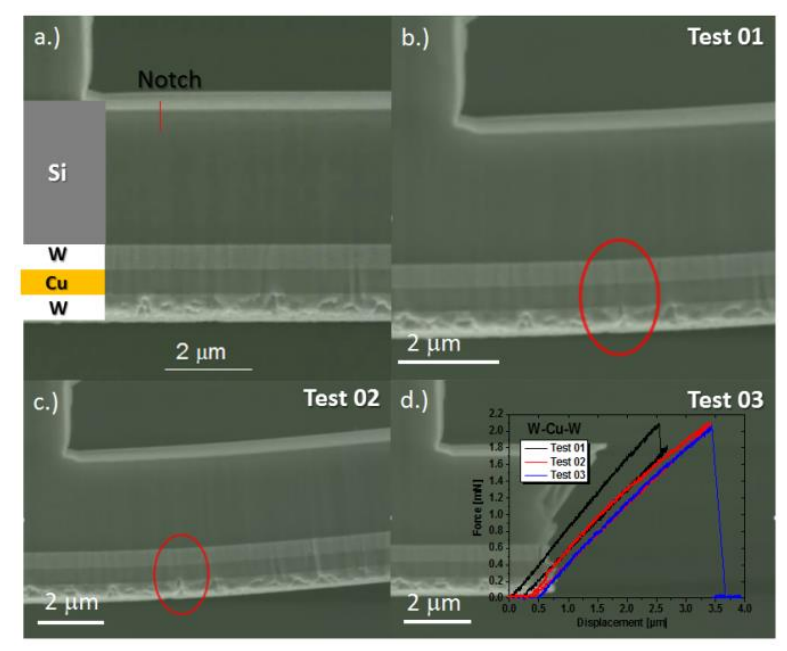

Figure 2. SE micrographs for a W-Cu-W system using three loading steps. The force is applied (not shown) at a distance of about $20 \mu \mathrm{m}$ from the notch. (a) Non-loaded sample. Notch is highlighted. (b) First test loading cycle, Test 01: crack (highlighted with circle) stops at the interface, (c) second test cycle, Test 02: possible crack extension in the $\mathrm{Cu}$ layer, (d) third test cycle, Test 03: ultimate fracture and force displacement data. For test 1 (black: upper curve), 2 (red: middle) and 3 (blue: lower curve). 